Rev. Ter. Ocup. Univ. São Paulo, v. 20, n. 2, p. 68-74, maio/ago. 2009.

\title{
Fatores que influenciam a escolha da área de atuação entre formandos em Terapia Ocupacional*
}

\section{Influencing factors on Occupational Therapy graduating' decision of working area}

\author{
Adriana de França Drummond ${ }^{1}$, Marisa Cotta Mancini², \\ Kátia Maria Penido Bueno ${ }^{3}$, Karina Righi Klausing ${ }^{4}$, \\ Leandro Barbosa de Moura ${ }^{5}$
}

\begin{abstract}
DRUMMOND, A. de F.; MANCINI, M. C.; BUENO, K. M. P.; KLAUSING, K. R.; MOURA, L. B. de. Fatores que influenciam a escolha da área de atuação entre formandos em Terapia Ocupacional. Rev. Ter. Ocup. Univ. São Paulo, v. 20, n. 2, p. 68-74, maio/ago. 2009.

RESUMO: Os objetivos deste estudo foram identificar os fatores que influenciam a escolha da área de atuação profissional de graduandos de Terapia Ocupacional da Universidade Federal de Minas Gerais (UFMG) e da Faculdade de Ciências Médicas de Minas Gerais (FCMMG); caracterizar a relevância desses fatores na escolha da área de atuação profissional e comparar fatores específicos da escolha de área de atuação profissional de graduandos do curso de Terapia Ocupacional em duas instituições da cidade de Belo Horizonte/MG, de naturezas distintas (pública e privada). Foi aplicado um questionário a todos os formandos do segundo semestre de 2001, primeiro e segundo semestres de 2002 da UFMG e às duas turmas de formandos de 2001 e 2002 da FCMMG. Os dados obtidos foram analisados de forma quantitativa por meio de frequencia. Os resultados revelaram como fatores relevantes (peso acima de 7/10) para o processo de escolha: gosto, vocação, preparo, relação com profissionais, experiências práticas curriculares, qualificação docente, relação professor/aluno e consistência teórico-clínica da área. Os resultados apresentados podem contribuir para que os cursos compreendam as influências que exercem sobre os formandos e possam, a partir daí, criar estratégias para a resolução de problemas.
\end{abstract}

DESCRITORES: Terapia Ocupacional. Escolha da profissão. Currículo.

\footnotetext{
* Apresentação do trabalho em eventos: Pôster no VIII Congresso Brasileiro de Terapia Ocupacional - Foz do Iguaçu, Paraná, Brasil, 2003.

${ }^{1}$ Mestre em Educação - Docente do Curso de Terapia Ocupacional daUniversidade Federal de Minas Gerais (UFMG).

2 Doutora em Ciências - Docente do Curso de Terapia Ocupacional da Universidade Federal de Minas Gerais (UFMG).

3 Doutora em Educação - Docente do Curso de Terapia Ocupacional da Faculdade de Ciências Médicas de Minas Gerais (FCMMG) e da Universidade FUMEC.

4 Terapeuta ocupacional - CREFITO $4^{a}$ Região Terapeuta ocupacional - CREFITO $4^{\circ}$ Região.

5 Acadêmico do Curso de Fisioterapia da UFMG.

Endereço para correspondência: Adriana Drummond - Rua São Romão 369/501, bairro Santo Antônio, CEP: 30330 -130 - Belo Horizonte/ MG, Brasil. e-mail: drummond@ufmg.br
} 


\section{INTRODUÇÃO}

$\mathrm{O}$ presente estudo estabeleceu como objetivos: identificar os fatores que influenciam a escolha da área de atuação profissional de graduandos de Terapia Ocupacional da Universidade Federal de Minas Gerais (UFMG) e Faculdade de Ciências Médicas de Minas Gerais (FCMMG); caracterizá-los em relação à relevância que exercem na escolha da área de atuação profissional e comparar fatores específicos da escolha da área de atuação profissional entre os graduandos de dois cursos de Terapia Ocupacional de naturezas distintas (pública e privada) localizados na cidade de Belo Horizonte/MG.

Segundo Hahn (2002, p. 3), "na literatura nacional específica de Terapia Ocupacional, pesquisada ao longo dos últimos 25 anos, não encontramos até a presente data nenhum trabalho que reflita e/ou discuta a questão das opções de áreas de especialização ou os seus equivalentes, seja ao longo do curso de graduação ou após a conclusão do mesmo". Em decorrência da carência de referências bibliográficas nacionais sobre a temática foram utilizados, prioritariamente, trabalhos realizados e divulgados no idioma inglês.

A revisão da literatura internacional na área da Terapia Ocupacional mostra estudos que enfatizam fatores determinantes na escolha da área de atuação de estudantes e em quais momentos do curso eles são relevantes. A decisão sobre se especializar em uma área prática pode ser influenciada por vários fatores, incluindo valores pessoais e personalidade, estilo de educação e aprendizagem, experiências clínicas de estágio, mercado de trabalho e família.

Wittman et al. (1989) investigaram mudanças de preferências pela prática em cinco momentos selecionados durante a formação profissional, a saber: 1) antes da admissão, 2) depois de completar o programa de estudo; 3) depois de completar o primeiro nível de estágio; 4) depois de completar o segundo nível de estágio; 5) no primeiro emprego. Segundo esses autores, as mudanças foram consideradas pequenas, tendo a porcentagem dos participantes interessados em determinada área no momento da admissão na graduação permanecido basicamente a mesma do momento da escolha do emprego.

Experiências de estágio também foram vistas como fortes fatores de influência na escolha da área de atuação profissional. O estágio introduz o estudante em uma gama de diferentes áreas práticas e uma variedade de settings no currículo. Isso lhe dá a chance de confirmar seu interesse em uma área e avaliar seu nível de habilidade clínica.

Tompson e Ryan (1996) estudaram as perspectivas de estágio em quatro estudantes do último ano do curso de Terapia Ocupacional e constataram que ele serviu para validar o conhecimento acadêmico. Os estudantes compararam a teoria com o que viviam na prática e puderam refletir sobre a relevância do trabalho acadêmico. Além disso, disciplinas ministradas em sala, consideradas pelos estudantes como irrelevantes e tediosas, foram avaliadas diferentemente dentro de um contexto clínico.

Outros fatores relacionados às experiências de estágio foram encontrados no estudo de Crowe e Mackenzie (2002), no qual o interesse pessoal na área, o retorno positivo dos estágios e o sentimento de confiança com o nível de habilidade requerido para determinada área foram as principais razões para a escolha da especialidade. Os participantes desse estudo relataram que é no estágio que se encontra a oportunidade de desenvolverem seus próprios valores de trabalho, tais como: 1) reconhecimento profissional; 2) alcance de senso de competência pessoal; 3) reconhecimento de clientes e da sociedade; 4) sensação de fazer a diferença como terapeuta ocupacional. Os supervisores de estágio também foram citados como influentes na preferência dos discentes pela área de prática. Três aspectos chaves do processo de supervisão foram ressaltados: 1) incentivo dos supervisores; 2) relação supervisor-aluno; 3 ) atitude profissional do supervisor com seu próprio trabalho.

Christie et al. (1985), em pesquisa com 188 terapeutas ocupacionais, supervisores e 127 estudantes em 65 campos de estágio sugerem que o campo de estágio tem significativo impacto no desenvolvimento da preferência de uma área específica da Terapia Ocupacional.

\section{MATERIAL E MÉTODOS}

Trata-se de um estudo observacional transversal de natureza descritiva e exploratória, visto a escassa produção acadêmica na temática. No início do ano de 2001 , as docentes deste projeto, envolvidas com o processo de reforma curricular do Curso desencadeado pela implantação das Diretrizes Curriculares Nacionais do Curso de Graduação em Terapia Ocupacional, questionaram-se sobre vários aspectos da formação do profissional no que concerne ao período de conclusão de Curso, o que desencadeou a referida pesquisa neste período.

Foram coletados dados por questionários semiestruturados para proceder-se à análise dos fatores que influenciam a escolha da área de atuação pelos formandos em Terapia Ocupacional da UFMG e FCMMG nos anos de 2001 e 2002.

A) Participantes: a amostra, não-aleatória, foi 
composta de 87 formandos de Terapia Ocupacional, sendo 45 da UFMG e 42 da FCMMG, assim distribuídos:

-27 formandos / FCMMG - ano 2001 (total de formandos: 30);

-20 formandos / UFMG - $2^{\circ}$ sem./2001 (total de

formandos: 21);

-11 formandos / UFMG - $1^{\circ} \mathrm{sem} . / 2002$ (total de formandos: 12);

-14 formandos / UFMG - $2^{\circ}$ sem./2002 (total de formandos: 15);

-15 formandos / FCMMG - ano de 2002 (total de formandos: 18).

Cabe ressaltar que o ingresso dos estudantes na UFMG é semestral e na FCMMG é anual.

B) Instrumentação: foi elaborado um questionário semi-estruturado para coleta de dados do estudo, contendo cinco perguntas, sendo três fechadas, uma mista e uma aberta. Esse instrumento incluiu informações sobre a área de escolha de atuação, o momento em que se fez a opção e fatores que influenciaram a escolha da área, especificando: gosto, preparação, identificação/proximidade com terapeutas ocupacionais e profissionais de áreas afins, expansão do mercado de trabalho, possibilidade de absorção no mercado de trabalho, possibilidade de retorno financeiro, influência de estágios curriculares e extracurriculares, consistência teórico-clínica da área, consolidação da área no curso, identificação com professores da área, qualificação dos docentes, influência do trabalho de conclusão de curso, influência de familiares e terceiros. Para as questões acerca dos fatores que influenciaram a escolha da área de atuação, os formandos identificaram um número entre zero (nenhum peso) e dez (peso máximo), pontuando, assim, o peso do fator na escolha da área de atuação. No final do questionário foi incluída uma questão aberta sobre comentários acerca da pontuação dos fatores acima.

C) Procedimentos: anteriormente ao início da coleta dos dados, foi realizado teste-piloto com quatro formandos dos cursos de Terapia Ocupacional da UFMG e da FCMMG para avaliar a adequação, clareza e pertinência das informações contidas no questionário em relação aos objetivos do estudo. Em seguida, e após esclarecimentos sobre a natureza do estudo, o questionário foi entregue e aplicado aos formandos pelos pesquisadores e por voluntários devidamente treinados, em horário previamente agendado com as turmas em salas de aula dos dois cursos, com orientação para preenchimento.

D) Análise dos dados: as informações obtidas pelos questionários foram analisadas de forma descritiva, indicando porcentagem de respostas dadas por estudantes dos cursos da UFMG e FCMMG nas diferentes categorias temáticas. Para melhor visualização, alguns resultados foram apresentados em gráficos.

\section{RESULTADOS}

Inicialmente, apresenta-se a caracterização geral da escolha da área de atuação (preferências de áreas de atuação e o momento do curso em que o formando fez essa escolha) e, em seguida, destacam-se os fatores de maior e menor relevância para escolha da área de atuação, respectivamente.

Os fatores foram agrupados em categorias temáticas similares, com o objetivo de facilitar a visualização de suas influências na escolha da área. Assim, a categoria "referências pessoais" englobou aqueles relacionados à influência familiar, ao gosto, vocação e identificação/ proximidade com terapeutas ocupacionais e profissionais de áreas afins. A categoria "estrutura curricular" envolveu fatores relacionados aos estágios curriculares, atividades extracurriculares, trabalho de conclusão de curso. A categoria "configuração da área" englobou aspectos de consistência teórico-prática e consolidação da área. A categoria "corpo docente" envolveu itens referentes à qualificação docente e relação professor/estudante. Finalmente, a categoria "mercado de trabalho" abrangeu aspectos relacionados à expansão e à absorção do profissional no mercado de trabalho e possibilidade de retorno financeiro.

\section{Caracterização geral da escolha}

Nas duas instituições de ensino superior, a maioria dos formandos já havia feito a escolha da área de atuação profissional antes do término da graduação, conforme apresentado no Gráfico 1.

GRÁFICO 1. Porcentagem de alunos da UFMG e FCMMG segundo a escolha da área antes do término da graduação

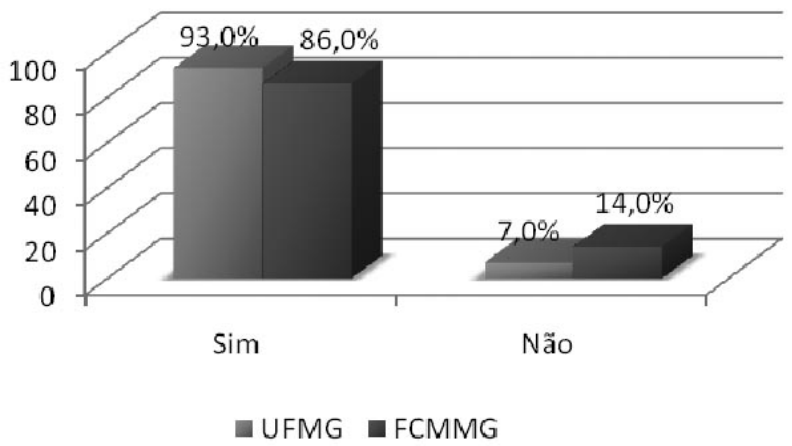


Essa escolha é feita prioritariamente a partir do contato do estudante com as disciplinas aplicadas em Terapia Ocupacional e com os estágios oferecidos pelas duas instituições de ensino superior, devendo-se destacar que $23 \%$ e $14 \%$ dos alunos da UFMG e da FCMMG, respectivamente, escolheram a área antes de ingressarem no Curso, conforme Gráfico 2.

GRÁFICO 2. Porcentagem de alunos da UFMG e FCMMG, segundo momento de escolha da área de atuação

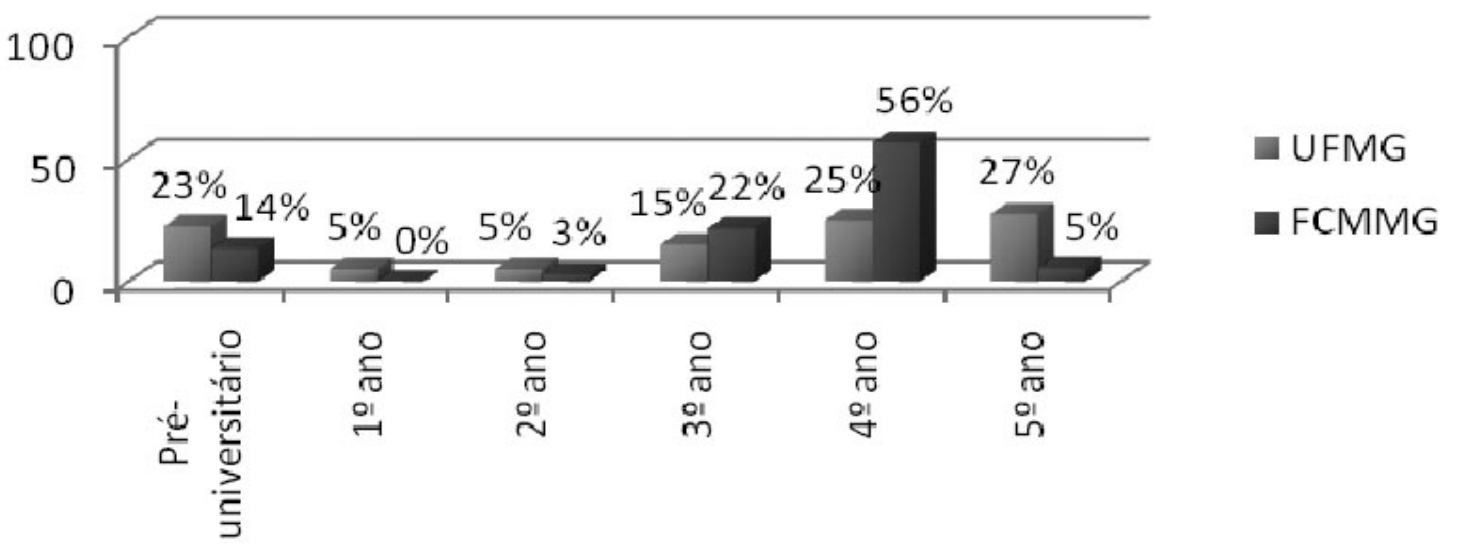

A reabilitação física foi a área de maior concentração de escolhas, quando a UFMG e a FCMMG foram analisadas em conjunto. Porém, houve preferência pela saúde mental

nesta e pelo desenvolvimento infantil naquela instituição, conforme exposto no Gráfico 3.

GRÁFICO 3. Porcentagem de Alunos da UFMG e da FCMMG segundo área de atuação escolhida

- UFMG $\square$ FCMMG

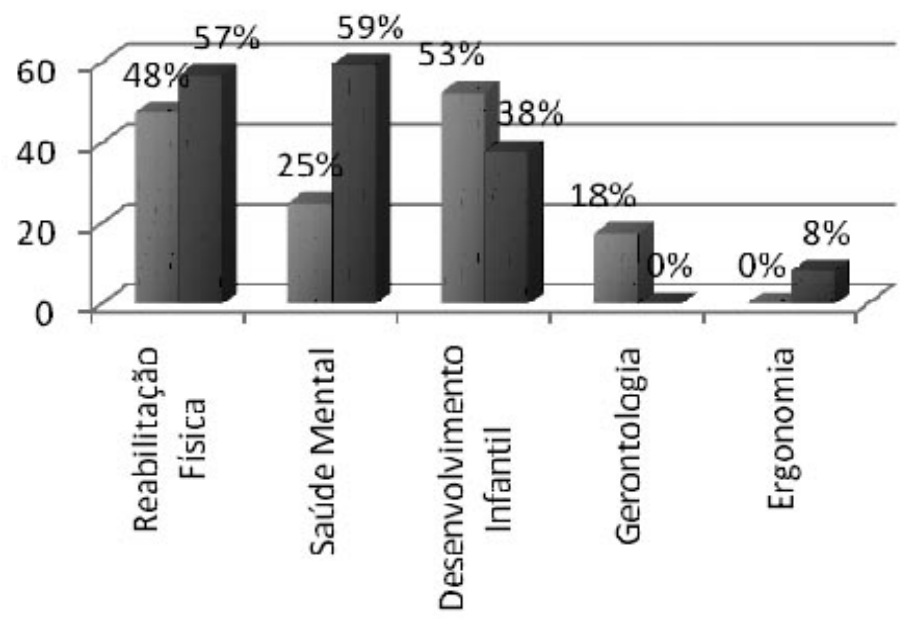

\section{Fatores relevantes para a escolha da área de atuação}

Sobre os fatores que influenciaram a escolha da área pelos formandos, não foram observadas diferenças significativas entre os formandos da UFMG e da FCMMG $(\mathrm{p}>0,05)$.

Conforme apresentado no Gráfico 4, ressalta-se o gosto pela área como elemento de maior relevância para a escolha da área de atuação do formando em Terapia Ocupacional da
UFMG e FCMMG, apresentando peso maior que nove.

Os estágios curriculares também se destacam como fator de grande relevância em ambas as instituições, com peso acima de oito, conforme salientado no Gráfico 4. Evidencia-se, ainda, a consistência teórico-prática da área com peso em torno de oito. Cabe ainda enfatizar o peso maior que sete pontuado tanto para a relação dos estudantes com os professores quanto para a qualificação docente em ambas as instituições. 
GRÁFICO 4. Peso atribuído pelos alunos da UFMG e FCMMG aos fatores relacionados à escolha da área de atuação

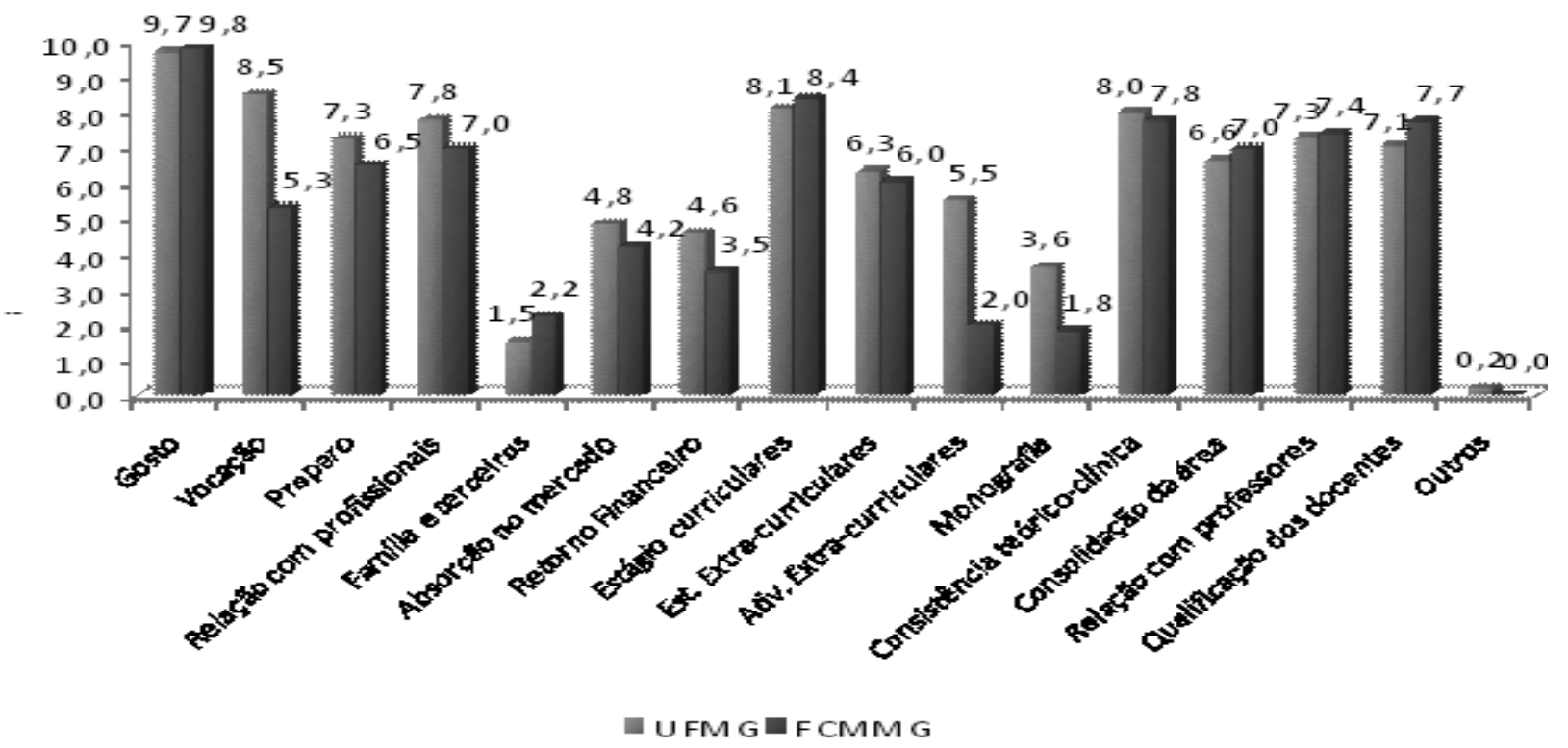

Fatores menos relevantes para a escolha da área de atuação

Os fatores relacionados com a expansão e a absorção do profissional no mercado de trabalho e a possibilidade de retorno financeiro mostraram-se insignificantes no processo de escolha de área de atuação pelos formandos tanto da UFMG quanto da FCMMG, bem como a influência de terceiros e a realização de trabalho de conclusão de curso (monografia), conforme indicado no Gráfico 4.

\section{DISCUSSÃO}

O gosto caracteriza-se como fator de primeira ordem de grandeza na influência da escolha da área de atuação pelos formandos da UFMG e FCMMG. E é ainda mais significativo entre aqueles que disseram ter escolhido a área antes do ingresso na graduação.

Segundo Bourdieu (1998) e Lahire (2002), o que está muitas vezes embutido na noção de gosto - quando a utilizamos como referência para qualificarmos as relações com objetos, situações, ações e práticas específicas - são preferências, interesses e certa tonalidade de relação, indicando um modo de apreciação. Esses autores relatam, ainda, que nossos gostos são ajustados às disposições constituídas a partir de nossas condições de existência e dos múltiplos processos socializadores pelos quais passamos e relacionam-se aos valores, princípios éticos e estéticos, estilos e esquemas de percepção, ação e pensamento. Exprimem, portanto, disposições que podem ter sido desenvolvidas e constituídas previamente ou ao longo do processo de formação.

O fator gosto é, neste estudo, potencializado pelo estágio curricular. Segundo relato dos formandos, "é na prática que se vê" ("Questionário número 5"), assim o gosto é reforçado ou negado nos estágios curriculares.

Lahire (2002) faz também referência aos termos vocação e dom, dizendo que, por sua vez, estes podem embutir a percepção de correspondência ou adequação entre as disposições possuídas e as situações de vida.

Observou-se que alunos da FCMMG tenderam a optar pela área de saúde mental enquanto alunos da UFMG pela área de desenvolvimento infantil. Tal tendência na porcentagem de preferência dos alunos pode estar atrelada aos fatores como área de capacitação docente, consolidação da área entre o corpo docente da instituição de ensino, possibilidades de pesquisa e qualidade dos estágios curriculares. Entretanto, o foco do presente estudo está voltado para identificação dos fatores que levam os alunos de ambas as instituições a escolher uma área de atuação profissional. Futuras investigações poderão avançar nos resultados apresentados, no que se refere aos mecanismos específicos de escolha das diferentes áreas de atuação em Terapia Ocupacional.

Pode-se discutir se o peso atribuído ao estágio tem relação com uma possível precariedade na articulação entre teoria e prática historicamente vivida nos cursos de graduação em Terapia Ocupacional. Como a oportunidade 
de conhecimento prático é reduzida ao longo dos cursos, concentra-se nas disciplinas aplicadas e nos estágios o peso da compreensão da realidade profissional, momento que corresponde aos quarto e quinto anos dos cursos, conforme apontado no Gráfico 2. A estrutura curricular dos dois cursos naquela época, estava ainda apoiada no Currículo Mínimo vigente para os Cursos de Terapia Ocupacional, o qual previa a organização curricular por ciclos, sendo o quarto e quinto anos correspondentes ao ciclo profissionalizante e aos estágios supervisionados, respectivamente.

Já os estágios extracurriculares configuram-se como a oportunidade de o estudante fazer escolhas pelo seu próprio interesse, às vezes impossibilitado pela extensa carga horária dos cursos, que dificulta a disponibilidade de tempo livre para a realização de atividades dessa natureza.

Pode-se também discutir se o peso mínimo atribuído aos fatores relacionados com expansão do mercado de trabalho, possibilidade de absorção no mercado de trabalho, possibilidade de retorno financeiro está relacionado aos aspectos de gênero, visto que a grande maioria da amostra é composta de terapeutas ocupacionais do sexo feminino. Lemel e Roudet (1999) avaliaram as socializações diferenciadas quanto ao aspecto do gênero, ressaltando as diferentes expectativas quanto às carreiras profissionais. Esses autores referiram que, geralmente, as escolhas femininas são mais fortemente orientadas pelos aspectos da expressividade pessoal, gosto e realização, enquanto as escolhas masculinas consideram aspectos mais concretos dos campos profissionais e se mostram preocupados com o objetivo de "ganhar a vida".

\section{CONCLUSÃO}

Podemos considerar que o gosto pela área de atuação é relevante para a escolha da área de atuação do formando em Terapia Ocupacional. A análise de gosto requer aprofundamento em questões como os múltiplos processos socializadores com os quais estamos envolvidos, desfazendo-se assim, a visão de que o gosto é um dado natural.

Investigações futuras devem analisar a relação entre escolha de áreas de atuação e gênero, visto que, para a profissão de Terapia Ocupacional, essa relação pode manifestar-se de forma reveladora.

Durante a graduação, as escolhas de área de atuação são prioritariamente feitas nos períodos relacionados com maior vivência prática, localizada nos quarto e quinto anos dos cursos, o que mostra, assim, o peso que os conteúdos aplicados e as práticas têm na formação do profissional.

Os dados obtidos neste estudo de natureza exploratória sugerem a relevância da análise das diferenças nas escolhas das áreas na UFMG e FCMMG em posteriores estudos longitudinais com este fim.

A identificação dos fatores que influenciam a escolha da área de atuação profissional entre formandos de cursos de graduação em Terapia Ocupacional contribui para que os cursos compreendam as influências que exercem sobre os discentes e a partir daí possam criar estratégias para a resolução de problemas que dificultam a formação do terapeuta ocupacional.

DRUMMOND, A. de F.; MANCINI, M. C.; BUENO, K. M. P.; KLAUSING, K. R.; MOURA, L. B. de. Influencing factors on Occupational Therapy graduating' decision of working area. Rev. Ter. Ocup. Univ. São Paulo, v. 20, n. 2, p. 68-74, maio/ago. 2009.

\begin{abstract}
The objectives of this study were to identify factors that influence the choice of professional acting area among Universidade Federal de Minas Gerais (UFMG) and Faculdade de Ciências Médicas de Minas Gerais (FCMMG) Occupational Therapy undergraduate students; to characterize the relevance of these factors in the choice of professional interest area and to compare specific choosing factors among the undergraduate students from both distinct (public and private schools) Occupational Therapy courses in the city of Belo Horizonte/MG. Surveying forms were distributed to all UFMG students graduating in the second semester of 2001, first and second semesters of 2002, and to the FCMMG graduating classes of 2001 and 2002. The data were analyzed with quantitative procedures by means of their statistical frequency. The results revealed the following factors as relevant, which were ranked higher (above 7 in 10) in their process of decision: fondness, vocation, readiness, relationship with professionals, curricular practical experience, faculty educational background, professor/student relationship, and area theoretical/clinical consistency. The results from this study may contribute to understanding the influences that these courses have on the graduating students and consequently, help to create problem solving.
\end{abstract}

KEY WORDS: Occupational therapy. Career choice. Curriculum. 
DRUMMOND, A. de F. et al. Fatores que influenciam a escolha. Rev. Ter. Ocup. Univ. São Paulo, v. 20, n. 2, p. 68-74, maio/ago. 2009.

\section{REFERÊNCIAS}

BOURDIEU, P. Escritos de educação. Petrópolis: Vozes, 1998.

CHRISTIE, B. A.; JOYCE, P. C.; MOELLER, P. L. Fieldwork experience, part I: impact on practice preference. Am. J. Occup. Ther, v. 39, n. 10, p. 671-674, 1985.

CROWE, M.; MACCKENZIE, L. The influence of fieldwork on the preferred future practice areas of final year occupational therapy students. Aust. Occup. Ther. J., v. 49, p. 25-36, 2002.

HAHN, M. S. Factors and reasons that influence clinical specialty choice among occupational therapist. In: CONGRESSO MUNDIAL DE TERAPIA OCUPACIONAL, 13, Suécia, 2002. Anais.... Suécia: Publicação eletrônica em CD-Rom. Australia: World Federation of Occupational Therapists, 2002.
LAHIRE, B. O homem plural: os determinantes da ação. Petrópolis: Vozes, 2002.

LEMEL, Y.; ROUDET, B. Filles et garcons jusqu'à l'adolescence: socializations différentielles. Paris: L'Harmattan, 1999. p.84127.

TOMPSON, M.; RYAN, A. Students' perspective of fielwork: process, purpose and relationship to coursework. Aust. Occup. Ther. J., v. 43, p. 95-104, 1996.

WITTMAN, P., et al. Variables affecting specialty choice in occupational therapy. Am. J. Occup. Ther., v. 43, n. 9, p. 602606, 1989. 